\title{
Relaxation dynamics of the mass-selected hydrated Auride ion ( $\mathrm{Au}$ )
}

\author{
Christian Braun ${ }^{a}$, Sebastian Proch ${ }^{\mathrm{a}, *}$, Young Dok Kim ${ }^{\mathrm{b}, *}$, Gerd Ganteför ${ }^{\mathrm{a}}$ \\ a Department of Physics, University of Konstanz, D-78457 Konstanz, Germany \\ ${ }^{\mathrm{b}}$ Department of Chemistry, Sungkyunkwan University, 440-746 Suwon, South Korea
}

\begin{abstract}
A B S T R A C T
By using time resolved photoelectron spectroscopy, decay of optically excited states of hydrated Au mass selected in the gas phase was studied. It is suggested that the pump photon can induce injection of an electron from auride chromophore to the water solvent. The cluster with three water molecules (Au $\left.\left(\mathrm{H}_{2} \mathrm{O}\right)_{3}\right)$ showed a long living excited state with a lifetime of $\sim 19 \pm 3 \mathrm{ps}$, whereas that of $\mathrm{Au}\left(\mathrm{H}_{2} \mathrm{O}\right)_{2}$ is only $\sim 3 \pm 1 \mathrm{ps}$. The fate of an optically excited state of an ionic species can be significantly changed by the number of water (solvent) molecules attached.
\end{abstract}

\section{Introduction}

The most important step in photochemical reactions is optical excitation of an electron from an occupied to an unoccupied state of a chromophore by absorption of light. When lifetimes of elec trons in optically excited states are long enough, initiation of a photochemical reaction can become competitive to recombina tion processes of electron hole pairs [1]. Femtosecond time re solved photoelectron spectroscopy (fsTRPES) has been widely used for shedding light on the relaxation dynamics and lifetimes of optically excited states [2]. The pump pulse excites an elec tron, whose relaxation process can be monitored by using a probe pulse with adjusted pump probe delay in fs time scale. In general, metallic species were found to show very fast relaxa tion of the optically excited state within several tens of fs via electron electron scattering mechanisms, whereas semiconduc tors exhibit much slower relaxation due to their absent density of states at the Fermi level [3].

Relaxation dynamics of small clusters consisting of less than $\sim 20$ atoms have been of considerable interest during the past dec ades. Particularly, mass selected clusters were used to study size dependent changes of relaxation dynamics of optically excited states of such small clusters on the atom by atom basis. For $\mathrm{d}$ me tal clusters, small clusters with less than 10 atoms already showed surprisingly short relaxation times (10 $100 \mathrm{fs}$ ) which are close to the respective values of bulk metals via electron electron scatter ing; since the whole valence $d$ shell electrons are donated to the cluster valence orbitals, the density of states of such small d metal clusters is high enough for allowing fast relaxation by electron electron scattering [ 4 6]. For sp metal clusters, in which relaxation of the excited state by electron electron scattering is not likely, the

\footnotetext{
* Corresponding authors.

E-mail addresses: Sebastian.proch@uni-konstanz.de (S. Proch), ydkim91@skku. edu (Y.D. Kim).
}

situation was found to be complex; for some clusters, lifetimes of excited states were very long (ns or ps time scale), whereas for others, efficient energy transfer from excited states to the nuclear motions of clusters allowed fs time scale relaxation dynamics $[7,8]$.

Not only bare metallic clusters but also metal clusters reacting with small molecules such as $\mathrm{CO}$ and $\mathrm{H}_{2} \mathrm{O}$ have been studied using fsTRPES [9 12]. As a function of the number of molecules attached to a metal cluster, relaxation dynamics were shown to vary signif icantly. Using fsTRPES we recently showed that for $\mathrm{Au}_{3}$, photodis sociation gives rise to the formation of $\mathrm{Au}$ and $\mathrm{Au}_{2}$ (or $\mathrm{Au}$ and $\left.\mathrm{Au}_{2}\right)$ species, and an additional $\mathrm{H}_{2} \mathrm{O}$ molecule $\left(\mathrm{Au}_{3}\left[\mathrm{H}_{2} \mathrm{O}\right]\right)$ acceler ated the photodissociation by a factor of $70[9,13]$. On the other hand, when more than two $\mathrm{H}_{2} \mathrm{O}$ molecules were attached to this cluster, the photodissociation of $\mathrm{Au}_{3}$ was suppressed completely [9]. Here, we studied the relaxation dynamics of $\mathrm{Au}$ with 2 and 3 water molecules adsorbed in which photodissociation of the chromophore is impossible. This system provides an opportunity to investigate the lifetimes of excited states with different degrees of solvation of the cluster without running into the problem of inducing photodissociation of the solute ( $\mathrm{Au}$ ).

\section{Experimental}

Gold cluster anions are produced in a pulsed arc cluster ion source (PACIS) and mass selected using a time of flight (ToF) mass spectrometer [14]. The experimental set up is described in detail elsewhere [15]. Au $\left(\mathrm{H}_{2} \mathrm{O}\right)_{m}$ with $m=04$ are produced by intro ducing water into the PACIS [9]. A selected bunch of cluster anions is irradiated by two pulses generated in a femtosecond Ti:sapphire laser system. The first pulse (pump pulse) has a photon energy of $3.1 \mathrm{eV}$ and the second pulse (probe pulse) a photon energy of $1.55 \mathrm{eV}$. The instrumental time resolution is $240 \mathrm{fs}$ and was deter mined by measuring the cross correlation function of the pump and probe pulses. The kinetic energy of the detached electrons is 
measured using a 'magnetic bottle' type time of flight electron spectrometer [16]. The cluster temperature is estimated to be room temperature, as they spend sufficiently long time in a water cooled extender for thermalization. The pump pulse results in photoexcitation of a certain fraction of the anions in the bunch and the probe pulse detaches electrons from a fraction of the anions in the same bunch. The photon flux of both pulses has to be optimized in order to maximize the number of species hit by a single photon of each pulse, and to minimize the number of spe cies hit by two or more photons from the same pulse.

For collecting regular photoelectron spectroscopy (PES) data, a photon energy of $7.9 \mathrm{eV}$ was used. For these experiments, the same method as that of the time resolved study described above was used for the preparation of the mass selected $\mathrm{Au}\left(\mathrm{H}_{2} \mathrm{O}\right)_{m}$ clusters.

\section{Results and discussion}

Figure 1 shows photoelectron spectra of $\mathrm{Au}\left(\mathrm{H}_{2} \mathrm{O}\right)_{m}$ clusters with $m=0 \quad 6$. In a previous study, Au $\left(\mathrm{H}_{2} \mathrm{O}\right)_{m}$ with $m=02$ were studied using PES [17], and we extended studies of solvated Au up to 6 water molecules attached. For bare $\mathrm{Au}$ three peaks cen tered at $2.31,3.41$, and $4.89 \mathrm{eV}$, respectively, could be identified. With increasing number of water molecules attached a gradual shift of these peaks of $\mathrm{Au}$ to higher binding energies was ob served. The fact that there are only rigid shifts of electronic states appearing as a consequence of solvation implies that the chromo phore of $\mathrm{Au}\left(\mathrm{H}_{2} \mathrm{O}\right)_{m}$ clusters is indeed $\mathrm{Au}$, which quite weakly interacts with adsorbed water molecules. As the number of water molecules in a cluster increased from 0 to 2 , the width of the peaks in PES increased [17]. For example, the lowest binding energy peak of $\mathrm{Au}$ had a full width of the half maximum (FWHM) of $0.15 \mathrm{eV}$, and this value increased to 0.25 and $0.27 \mathrm{eV}$ as the number of water molecules in a cluster became 1 and 2 . For $m>2$, the FWHM of the first peak of each PES spectrum was $0.27 \pm 0.1 \mathrm{eV}$, i.e., as the number of water molecules in a cluster exceeded 2 , no further broadening of the peaks in the PES spectra was found.

As it has been shown previously, the PE spectral shift of the cluster by an additional water molecule can be used for approximately estimating the energy required to remove this addi tional water molecule from the anionic cluster, i.e. based on the PE spectra in Figure 1, the binding energy of water molecules in the clusters can be roughly estimated [17]. For bare Au , the electron affinity (EA) value is estimated to be $2.31 \mathrm{eV}$, and VDE of $\mathrm{Au}\left(\mathrm{H}_{2} \mathrm{O}\right)$ is found to be $2.76 \mathrm{eV}$, and based on these data the binding energy of the first water molecule attached to $\mathrm{Au}$ is estimated to be $0.45 \mathrm{eV}$. Note that the binding energy of $\mathrm{H}_{2} \mathrm{O}$ in $\mathrm{Au}\left(\mathrm{H}_{2} \mathrm{O}\right)$ can be estimated based on the following equation.

(VDE of $\mathrm{Au}\left(\mathrm{H}_{2} \mathrm{O}\right) \quad \mathrm{EA}$ of $\left.\mathrm{Au}\right) \quad \mathrm{D}_{0}\left(\mathrm{Au} \quad \ldots \mathrm{H}_{2} \mathrm{O}\right) \quad \mathrm{D}_{0}\left(\mathrm{Au} \ldots \mathrm{H}_{2} \mathrm{O}\right)^{*}$

$D_{0}\left(\mathrm{Au} \ldots \mathrm{H}_{2} \mathrm{O}\right)$ and $\mathrm{D}_{\mathrm{o}}\left(\mathrm{Au} \ldots \mathrm{H}_{2} \mathrm{O}\right)^{*}$ denote dissociation energies of $\mathrm{H}_{2} \mathrm{O}$...Au in $\mathrm{Au}\left(\mathrm{H}_{2} \mathrm{O}\right)$ and $\mathrm{Au}\left(\mathrm{H}_{2} \mathrm{O}\right)$ at the structure of $\mathrm{Au}\left(\mathrm{H}_{2} \mathrm{O}\right)$, respectively, and $\mathrm{D}_{0}\left(\mathrm{Au} . \ldots \mathrm{H}_{2} \mathrm{O}\right)^{*}$ can be regarded as very small and therefore neglected. For more details of the estimation of the bind ing energy of water molecules in the hydrated Au clusters, read ers should refer to Ref. [17]. With increasing number of water molecules, the spectral shift becomes less and less. The binding en ergy of the sixth water molecule is estimated to be less than $0.32 \mathrm{eV}$, which is only about $70 \%$ of the binding energy of the first water molecule.

Figure 2 displays the PE spectra of $\mathrm{Au}\left(\mathrm{H}_{2} \mathrm{O}\right)_{m}$ with $m=1$ to 4 , which were obtained using coincident pump and probe pulses with energies of $3.1 \mathrm{eV}$, and $1.55 \mathrm{eV}$, respectively, i.e. the pump probe time delay was 0 for obtaining the spectra in Figure 2. The binding energy here was determined to be the difference between the probe photon energy and the kinetic energy of photoelectrons. For $\mathrm{Au}\left(\mathrm{H}_{2} \mathrm{O}\right)$, a peak with a negative binding energy could be seen. The VDE of $\mathrm{Au}\left(\mathrm{H}_{2} \mathrm{O}\right)$ in Figure 1 was found to be $2.79 \mathrm{eV}$. The bind ing energy of the peak with the lowest binding energy of $\mathrm{Au}\left(\mathrm{H}_{2} \mathrm{O}\right)$ in Figure 2 is $0.31 \mathrm{eV}$, which is difference between VDE of $\mathrm{Au}$ $\mathrm{Au}\left(\mathrm{H}_{2} \mathrm{O}\right)(2.79 \mathrm{eV})$ and pump photon energy $(3.1 \mathrm{eV})$. Since the same pump photon energy $(3.1 \mathrm{eV})$ was used for coincident pump probe spectra of all clusters in Figure 2, the binding energy of the lowest binding energy peak of each cluster should be higher than that of the VDE of the respective cluster by $3.1 \mathrm{eV}$. As it will be seen later, these peaks in figures do not necessarily correspond to

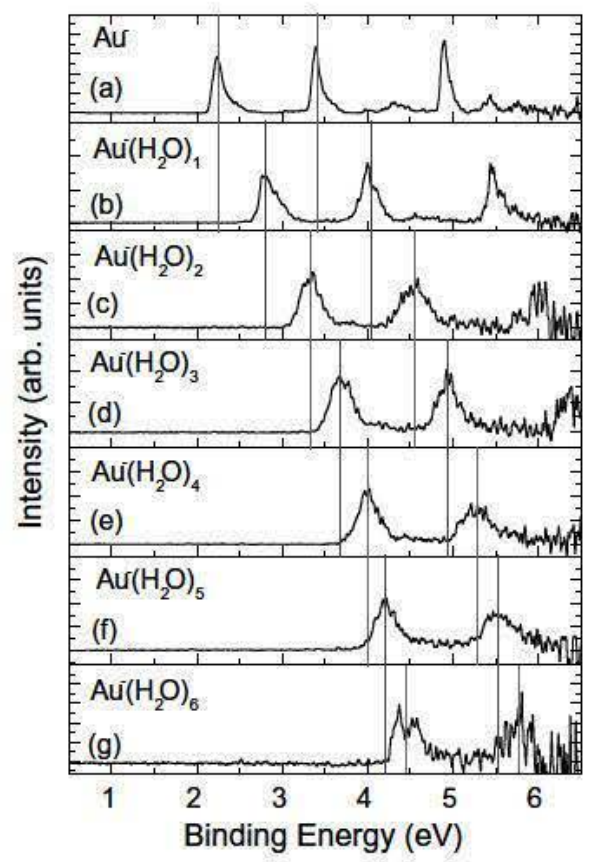

Figure 1. Photoelectron spectra of solvated auride ions with 0 to 6 water molecules. Center of each peak is denoted with vertical line.

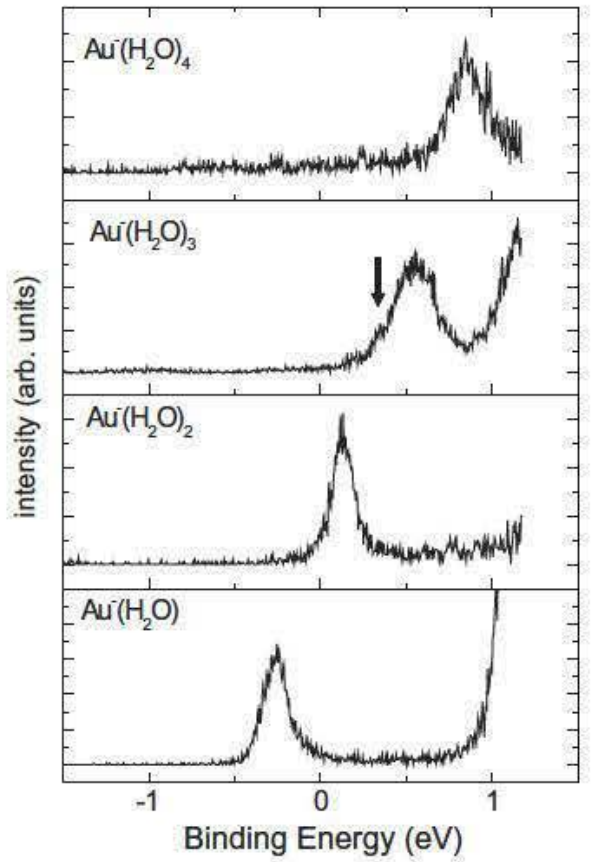

Figure 2. Pump-probe spectra of solvated auride ions with 1 to 4 water molecules at zero time delay. 
resonant excitations, thus non resonant excitations cannot be excluded.

In Figure 2, it is notable that the peak of PE spectrum of $\mathrm{Au}\left(\mathrm{H}_{2-}\right.$ O) ${ }_{3}$ is significantly broader than those of other clusters with smal ler or larger numbers of water molecules in a cluster. Particularly, a peak centered at $0.57 \mathrm{eV}$ and a shoulder at $0.37 \mathrm{eV}$ (marked with an arrow) can be seen in the photoelectron spectrum of $\mathrm{Au}\left(\mathrm{H}_{2} \mathrm{O}\right)_{3}$ using pump and probe with 0 delay. As it will be shown later, there are two different components here, which are resonant and non resonant excitations, respectively.

In order to shed light on the relaxation dynamics of the opti cally excited states of hydrated audride, fsTRPES of $\mathrm{Au}\left(\mathrm{H}_{2} \mathrm{O}\right)_{m}$ with $n=14$ were recorded. For $m=1$ and 4 , the peaks shown in Figure 2 at zero delay spectra rapidly disappeared with increasing pump probe delay; the intensity of these peaks can only be seen within the pump probe delay range of 250 to $250 \mathrm{fs}$ (data not shown). One can suggest that non resonant excitation by pump photon took place for $m=1$ and 4 . Only for $A u\left(\mathrm{H}_{2} \mathrm{O}\right)_{m}$ with $m=2$ and 3 , excited states survived for significantly longer times of pump probe delay compared to our upper limit of the time res olution. Therefore, it is suggested that only these clusters show resonant excitation using our pump photon energy ( $3.1 \mathrm{eV}$, Figures 3 and 4 ).

For $m=2$, the pump probe signal showed a single peak cen tered at the binding energy of only $0.1 \mathrm{eV}$, when the pump probe
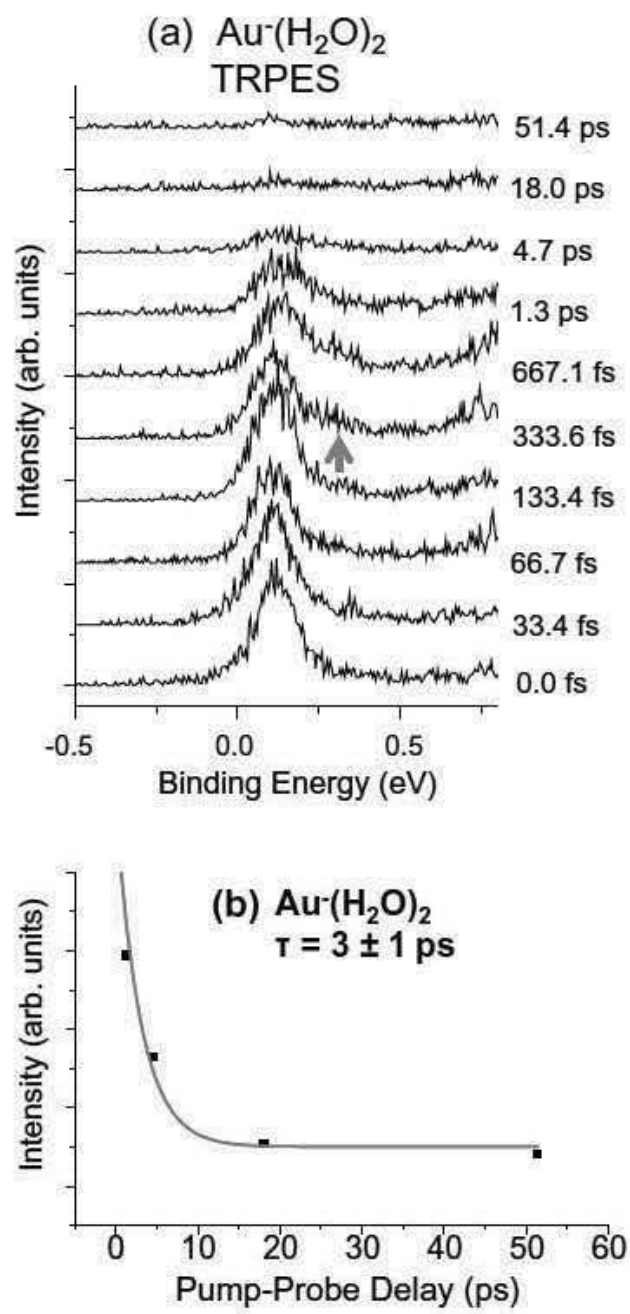

Figure 3. (a) Time-resolved photoelectron spectra for solvated auride ions with 2 water molecules. (b) Decay of the optically excited states of solvated auride ions with 2 water molecules is summarized. delay was shorter than $100 \mathrm{fs}$. When a longer pump probe delay was used, an additional state centered at $0.3 \mathrm{eV}$ appeared (de noted with arrow in the figure) whereas reduction of the inten sity of the peak at $0.1 \mathrm{eV}$ took place at the same time. When the pump probe delay became longer than $1.3 \mathrm{ps}$, a gradual de crease in the intensity of both pump probe peaks could be found. Since we could observe non resonant excitation for $m=1$ and 4 under the same conditions as those of TRPES experiment for $m=2$, non resonant excitation should also be seen for $m=2$. It is likely that the broad peak centered at $0.1 \mathrm{eV}$ corresponds to the overlap of non resonant and resonant excitation, whereas that at $0.3 \mathrm{eV}$ corresponds to purely resonant excitation. The peak at $0.1 \mathrm{eV}$ does not fully disappear with increasing pump probe delay and therefore, this peak cannot be assigned solely non res onant excitation.

Previous studies on I $\left(\mathrm{H}_{2} \mathrm{O}\right)_{m}$ clusters using TRPES found that the pump photon can excite an electron from the I chromophore to the surrounding water molecules, which is weakly bound with binding energies of several hundred $\mathrm{meV}$ [18]. The electron injected from the anionic chromophore to the surrounding water molecules should temporarily occupy an electronic state of water, which is weakly bound by the dipole moment of water molecules. The binding energies of the excited states assigned to the charge transfer to solvent (CTTS) in $\mathrm{I}\left(\mathrm{H}_{2} \mathrm{O}\right)_{m}$ are close to those of the pump probe signals of $\mathrm{Au}\left(\mathrm{H}_{2} \mathrm{O}\right)_{2}$. Therefore, it is reasonable to suggest that we have also CTTS in the Au $\left(\mathrm{H}_{2} \mathrm{O}\right)_{2}$ induced by the pump photon. In Figure $3 \mathrm{~b}$, the integrated intensity of the

(a) $\mathrm{Au}^{-}\left(\mathrm{H}_{2} \mathrm{O}\right)_{3}$ TRPES
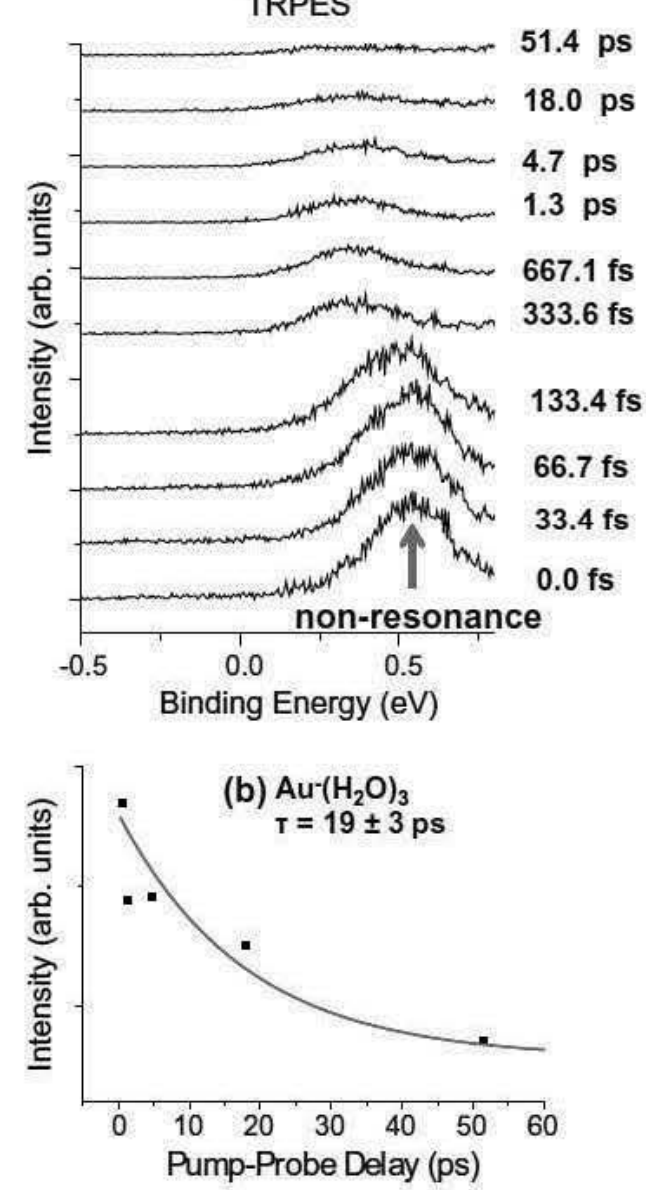

Figure 4. (a) Time-resolved photoelectron spectra for solvated auride ions with 3 water molecules. (b) Decay of the optically excited states of solvated auride ions with 3 water molecules is summarized. 
pump probe signal of $\mathrm{Au}\left(\mathrm{H}_{2} \mathrm{O}\right)_{2}$ in the pump probe delay range above $200 \mathrm{fs}$ was fitted as a function of pump probe delay by exponential relaxations. The lifetime of the slow relaxation of $\mathrm{Au}\left(\mathrm{H}_{2} \mathrm{O}\right)_{2}$ is estimated to be $\sim 3 \pm 1 \mathrm{ps}$. The fact that we observed a peak of excited state centered at $0.1 \mathrm{eV}$ for shorter pump probe delay and an additional peak at $0.3 \mathrm{eV}$ at longer ones is also in agreement with previous results of $\mathrm{I}\left(\mathrm{H}_{2} \mathrm{O}\right)_{m}$. Here, the initial drop in the binding energy of the excited state was attributed to the repulsive interaction between solvent and detached elec trons induced by the rapid geometric rearrangement of water molecules in the cluster just after photoexcitation by pump pulse $[18,19]$.

In the case of $\mathrm{Au}\left(\mathrm{H}_{2} \mathrm{O}\right)_{3}$, a major peak overlapped with a shoul der at lower binding energy appeared in the zero delay spectrum and the peak centered at $0.57 \mathrm{eV}$ disappeared rapidly as a function of pump probe delay, whereas that at $0.37 \mathrm{eV}$ relaxed more slowly. Here again, the peak centered at $0.37 \mathrm{eV}$ can be attributed to the CTTS from auride to water solvent. The rapidly disappearing peak at binding energy of $0.57 \mathrm{eV}$ of Figures 2 and 4 can be attrib uted to the non resonant excitation, as we have also observed for $m=1$ and 4 . The decay curve of Figure $4 \mathrm{~b}$ shows that the lifetime of the excited state at $0.37 \mathrm{eV}$ is $\sim 19 \pm 3 \mathrm{ps}$, which is significantly longer than that for two water molecules. It was previously sug gested that the additional electron can be more stabilized by an increasing number of water molecules in a cluster, which can ulti mately result in a longer lifetime of the electron temporarily resid ing in water, in line with our results [18].

We observed resonant excitation only when two or three water molecules were attached to the auride ion. In water solvents an electron should be bound by a binding energy of several hundreds $\mathrm{meV}$ and the excited states corresponding to such weakly bound electrons appear at these binding energies. By using a fixed pump energy $(3.1 \mathrm{eV})$ such states can only be reached in the case of clusters with a suitable ground state electronic structure. There should be an electronic state with binding energy comparable to the sum of $3.1 \mathrm{eV}$ plus the binding energy of weakly bound elec tron at water ( $\sim$ several hundreds $\mathrm{meV}$ ).

A long living optically excited state can result in photochemis try, since only electrons at excited state with sufficiently long life time can participate in chemical reactions, in which transfer of optically excited electrons of the chromophore needs to be initi ated. For example, $\mathrm{TiO}_{2}$ photocatalysts can start chemical reactions by electron transfer from the $\mathrm{TiO}_{2}$ conduction band to $\mathrm{OH}$ groups formed during water splitting [1]. Here, we show that lifetime of excited electrons at solvated anion can be considerably changed by altering the number of solvents attached to the ion.

\section{Conclusion}

Using fsTRPES, the decay of optically excited states of solvated Au mass selected in the gas phase using ToF mass spectrometry was studied. Relaxation time was found to be very sensitive to the number of water molecules in a cluster: the cluster with three water molecules $\left(\mathrm{Au}\left(\mathrm{H}_{2} \mathrm{O}\right)_{3}\right)$ showed a long living excited state with a lifetime of $19 \pm 3 \mathrm{ps}$, whereas auride with two water mole cules attached showed a lifetime of $3 \pm 1$ ps for the optically ex cited state. We show that the lifetime of the optically excited state of an ionic species can be changed by the number of water molecules attached, implying that photochemistry initiated by such species can be strongly dependent on the degree of solvation.

\section{Acknowledgments}

Financial support from the German Research Foundation (DFG) Grant No. GA 389/12 2 is gratefully acknowledged. This work was supported by MEST and PAL, XFEL project, Korea.

\section{References}

[1] M. Kitano, K. Tsujimaru, M. Anpo, Top. Catal. 49 (2008) 4.

[2] A. Stolow, A.E. Bragg, D.M. Neumark, Chem. Rev. 104 (2004) 1719.

[3] K. Koyasu, C. Braun, S. Proch, G. Gantefoer, Appl. Phys. A 100 (2010) 431.

[4] N. Pontius, P.S. Bechthold, M. Neeb, W. Eberhardt, Phys. Rev. Lett. 84 (2000) 1132.

[5] N. Pontius, G. Luttgens, P.S. Bechthold, M. Neeb, W. Eberhardt, J. Chem. Phys, 115 (2001) 10479.

[6] N. Pontius, M. Neeb, W. Eberhardt, G. Luttgens, P.S. Bechthold, Phys. Rev. B 67 (2003) 035425.

[7] Y.D. Kim, M. Niemietz, P. Gerhardt, F. von Gynz-Rekowski, G. Gantefor, Phys. Rev. B 70 (2004) 035421.

[8] M. Niemietz, M. Engelke, Y.D. Kim, G. Gantefor, Phys. Rev. B 75 (2007) 085438.

[9] C. Braun, S. Proch, H.O. Seo, Y.D. Kim, G. Gantefor, Chem. Phys. Lett. 530 (2012) 35.

[10] G. Luttgens, N. Pontius, P.S. Bechthold, M. Neeb, W. Eberhardt, Phys. Rev. Lett. 88 (2002) 076102.

[11] K. Koyasu, M. Niemietz, W. Westhaeuser, G. Gantefoer, Eur. Phys. J. D 53 (2009) 59.

[12] K. Koyasu, W. Westhaeuser, M. Niemietz, J. Heinen, G. Gantefoer, Appl. Phys. A 96 (2009) 679.

[13] G. Ganteför, S. Kraus, W. Eberhardt, J. Electron Spectrosc. Relat. Phenom. 88 (1998) 35.

[14] G. Ganteför, H.R. Siekmann, H.O. Lutz, K.H. Meiwes-Broer, Chem. Phys. Lett. 165 (1990) 293.

[15] G. Ganteför, C.Y. Cha, W. Eberhardt, Rev. Sci. Instrum. 63 (1992) 5661.

[16] G. Ganteför, H. Handschuh, W. Eberhardt, Rev. Sci. Instrum. 66 (1995) 3838.

[17] W. Zheng, X. Li, S. Eustis, A. Grubisic, O. Thomas, H. de Clercq, K. Bowen, Chem. Phys. Lett. 444 (2007) 232.

[18] R.M. Young, D.M. Neumark, Chem. Rev. 112 (2012) 5553.

[19] C.C. Mak, Q.K. Timerghazin, G.H. Peslherbe, Phys. Chem. Chem. Phys. 14 (2012) 6257. 